\title{
SENS ET FORMES DANS LA SÉMANTIQUE MARRISTE
}

\author{
Ekaterina Velmezova ${ }^{1}$
}

Abstract: Object of several criticims but seldom read the sovietic linguist Nicolai Ja. Marr (1864-1934) let us a theorethical heritage which requests more complexes investigations. His approaches on identity articulated to semantics were produced around the notion of semantics'laws.

A detailed analysis on his semantics'laws can authorize us to elaborate a particular epistemic approach on Marr's heritage. This authorizes us to understand better the theory of Marr, not with standing his theory seems to be incomprehensible and illogical.

Key-words: identities' approaches, Marrism, evolutionism, semantics, law

Resumo: Muito criticado, mas pouco lido nas últimas décadas, o lingüista soviético Nicolai Ja. Marr (1864-1934) deixou-nos uma herança teórica que demanda investigações mais aprofundadas. Suas investigações semânticas, ligadas a abordagens identitárias, alicerçam-se na noção de lei semântica.

Uma análise detalhada das leis semânticas, por ele formuladas, nos remete à elaboração de uma abordagem epistemológica particular sobre a herança marrista. Isto nos permite compreender melhor a teoria de Marr, ainda que esta pareça incompreensível e ilógica à primeira vista.

Palavras-chave: abordagens identitárias, Marrismo, evolucionismo, semântica, lei.

\section{PROBLÈMES IDENTITAIRES DANS L'HÉRITAGE THÉORIQUE DE N. MARR}

Thématique et problématique, buts et matériaux de la présente recherche

Parmi tous les problèmes passionnants qui puissent intéresser les historiens de la linguistique, l'un nous semble particulièrement intéressant. Il s'agit de chercher à comprendre ce qui semble incompréhensible et illogique, voire incohérent à première vue. En particulier, la plupart des historiens de la linguistique traitent ainsi la doctrine linguistique de Nikolaj Jakovlevič Marr (1864-1934) - linguiste soviétique, créateur de la «nouvelle théorie du langage » (novoe učenie ob jazyke), connu aussi sous le nom de marrisme.

L'évolution même du marrisme en tant que théorie linguistique particulière est directement liée avec les problèmes identitaires. En fait, dans l'héritage linguistique de Marr on distingue traditionnellement deux périodes. La première période est celle de la théorie japhétique, selon laquelle il existerait une famille particulière de langues, la famille japhétique. Le géorgien, qui était la langue

\footnotetext{
${ }^{1}$ Université de Lausanne - Académie des sciences de Russie.
} 
maternelle de Marr ${ }^{2}$, y ferait partie. Déjà à l'école Marr s'est donné pour but de montrer la parenté de la langue géorgienne (dont les racines n'étaient pas encore bien étudiées à cette époque) avec les autres langues (en particulier, avec le turc), ce qui était directement lié avec ses quêtes identitaires. Pourtant en développant cette théorie, dans les années 1920 Marr a renoncé à la notion même de parenté linguistique et de familles de langues pour les remplacer par celles des stades dans le développement langagier. C'est cette (deuxième) partie de son héritage théorique qui a reçu le nom de marrisme.

Pendant la vie de Marr et après sa mort, sa théorie linguistique fit office de « doctrine officielle » dans la linguistique soviétique. Mais en 1950, lors de la discussion publique dans le journal Pravda, elle fut désapprouvée et renversée par J.V. Staline. Par la suite, les éloges immodérés à l'adresse de Marr furent très vite remplacés par des accusations nombreuses, en particulier celle de manque de valeur scientifique de ses théories. Dans une certaine mesure, cette situation continue jusqu'à nos jours, en se reflétant dans de nombreuses recherches consacrées à Marr, au marrisme et aux marristes. A la différence de la plupart des études sur Marr et le marrisme qui existent déjà, nous ne visons pas l'étude du marrisme ni en tant que tel, ni dans ses liens avec le régime politique en URSS dans les années 1920-1950, même si ce facteur extralinguistique ne pourra pas être complètement exclu de ce travail. Dans notre cas, la thématique ne fait que fournir le matériau nécessaire à l'étude de la problématique proprement dite, qui peut être définie comme l'étude d'une page particulière et peu connue de l'histoire de la sémantique. Autrement dit, l'étude du marrisme en tant que courant particulier de la linguistique soviétique des années 1920-1950 devra nous permettre de découvrir des faits jusqu'à maintenant inconnus ou très peu étudiés de l'histoire de la sémantique en Russie et en URSS à la fin du XIXème et au début du XXème siècles.

D'autre part, le marrisme est une doctrine qui semble si compliquée et contradictoire que, à notre avis, l'une des tâches des historiens de la linguistique pourrait consister précisément à chercher ou à élaborer une telle interprétation du marrisme qui permettrait, même en partie, de lever certaines de ces contradictions. La recherche de ce point de vue, ainsi que le travail dans le cadre de ce principe constituera le but de cette étude.

Dans notre travail, nous nous appuierons, avant tout, sur le matériau des sources primaires. Malgré toutes les difficultés de ce travail, nous sommes d'avis que la qualité de tout travail lié à l'histoire des idées linguistiques dépend de ce choix. Bien que l'étude d'un sujet aussi complexe que le marrisme puisse sembler être plus facile à travers les sources secondaires (les

${ }^{2}$ La mère de Marr était Géorgienne et son père était Ecossais. 
textes de Marr et de certains de ses élèves semblent parfois incompréhensibles même aux russophones), elle ne peut amener qu'à des erreurs et des malentendus.

En travaillant dans le cadre de l'épistémologie comparée, nous prêterons une attention particulière à l'analyse conceptuelle. La célèbre thèse des marxistes qui se sont intéressés au rapport entre langue et idéologie depuis M.M. Bakhtine et V.N. Vološinov jusqu'à L. Althusser et M. Pêcheux - sur la non-transparence des mots et sur leurs significations apparaissant plutôt dans la parole (ou dans le discours) que dans la langue, est d'autant plus pertinente quand il s'agit de textes scientifiques, où derrière les mots et même les termes anodins à première vue, des sens inattendus peuvent se cacher. Ce fait révèle toute l'importance, pour les historiens de la linguistique, d'analyser en détail la terminologie des travaux particuliers.

La direction générale de notre recherche est également à souligner. Dans la plupart des travaux sur le marrisme, sa composante purement linguistique est pratiquement toujours mélangée avec l'étude des événements extérieurs - il s'agit, avant tout, de la vie politique en URSS de cette époque. Nous n'ignorerons pas les événements extérieurs à la linguistique. En même temps, au lieu de leur attribuer une valeur explicative, nous essaierons, au contraire, de partir de la linguistique elle-même pour résoudre tel ou tel "mystère » extralinguistique, que ce soit une traduction erronée ou l'intervention d'un dictateur dans la science.

L'analyse des quatre lois sémantiques de Marr - loi de la «perception humaine commune », loi des opposés, loi de la sémantique diffuse et loi de la transposition fonctionnelle ${ }^{3}$ - nous servira de base de depart.

\section{LOIS SÉMANTIQUES CHEZ MARR}

Loi de la perception humaine commune, ou la mystère d'une traduction russe (La sémantique dépassant la forme)

En 1930, le premier livre en russe du sociologue et anthropologue français Lucien Lévy-Bruhl (1857-1939) parut en URSS ${ }^{4}$. Traduit en russe, ce livre avait pour titre Pervobytnoe myšlenie - ce qui théoriquement devait correspondre au titre français original La mentalité primitive. Et pourtant, sous le titre La mentalité primitive (Pervobytnoe myšlenie), c'est un tout autre livre qu'on avait traduit en russe...

\footnotetext{
${ }^{3} \mathrm{~A}$ la différence de nombreux chercheurs qui attribuent à Marr les découvertes sémantiques qu'il n'avait jamais fait, nous n'analyserons ici que ce que Marr lui-même traite de loi.

${ }^{4}$ Levi-Brjul' (Lévy-Bruhl), 1930.
} 
En fait, l'auteur était le même - c'était bien un livre de Lévy-Bruhl qu'on avait traduit. De plus, certains morceaux de La mentalité primitive avaient été inclus dans l'ouvrage traduit. Or il s'agissait de la traduction d'un autre livre - Les fonctions mentales dans les sociétés inférieures ${ }^{5}$, dont le titre aurait été traduit en russe tout à fait autrement: Mental'nye (myslitel'nye) funkcii $v$ ob̌šcestvax nižsego tipa.

L'éventualité d'une erreur du traducteur semble exclue: en fait, il est peu probable que quelqu'un soit distrait au point qu'il traduise un livre en lui donnant le titre d'un autre. Au contraire, nous sommes ici en présence d'une tentative réussie de substitution qui a été légitimée par une maison d'édition soviétique très respectée ( «Ateist ») et par des commissions bureaucratiques nombreuses. Bref, il s'agit d'une substitution, presque d'une fraude. Et nous voilà devant la question classique qui apparaît toujours dans semblable cas: Cui prodest? A qui profitait-il de cacher le titre authentique du livre traduit?

Essayons de comparer les deux titres originaux, La mentalité primitive et Les fonctions mentales dans les sociétés inférieures, pour comprendre pourquoi l'un des deux a été préféré à l'autre - au point que le traducteur «ait confondu » le titre du livre en russe. Dans ce contexte, les notions de « fonctions mentales » et «mentalité » semblent fonctionner de façon synonymique. Donc tout le problème est celui des adjectifs primitif et inférieur. Pourquoi a-t-on remplacé l'un par l'autre, dans la traduction russe?

Dans la tradition russe, la traduction de certains détails d'ouvrages de sciences humaines ne dépend pas toujours du traducteur. Très souvent, les décisions définitives sont celles du «rédacteur» ou de la personne qui choisit le contenu du livre et, ce qui arrive souvent dans ce cas, compose aussi la préface de l'ouvrage correspondant.

Dans notre cas, la préface a été composée par Marr. Cela signifie que même si ce n'était pas Marr en personne qui a changé le titre du livre traduit, il a dû cautionner cette substitution. Pourquoi l'a-t-il fait?

Pour répondre à cette question, commençons par une autre, plus simple: qu'est-ce qui a intéressé Marr dans les conceptions de Lévy-Bruhl? Pourquoi lui, tellement occupé, président et membre d'innombrables comités et commissions, auteur d'un grand nombre d'articles, a-t-il activement participé à la traduction du livre de Lévy-Bruhl en russe?

On pourrait supposer qu'il y avait quelque chose en commun dans les conceptions de Marr et de Lévy-Bruhl. Qu'est-ce qui rapprochait ces deux théories ${ }^{6}$ ?

\footnotetext{
${ }^{5}$ Lévy-Bruhl, 1910.

${ }^{6}$ Pour la réponse plus détaillée à cette question cf. Velmezova, 2003 et Velmezova, 2005a.
} 
En élaborant la théorie de la pensée "primitive» ou «inférieure », LévyBruhl affirmait que, pour l'essentiel, cette dernière se distinguait fondamentalement de la pensée dite moderne, ou rationnelle - une thèse contestant l'ethnocentrisme de l'Ecole anthropologique anglaise dont les représentants (E. Tylor, J. Frazer) partageaient l'axiome que l'esprit humain est toujours et partout identique à lui-même. Selon Lévy-Bruhl, «les primitifs ne perçoivent rien comme nous $\gg^{7}$.

La première des lois sémantiques de Marr est celle de la «perception humaine commune ». Marr en parle en particulier dans son article de 1926, dans le contexte suivant :

«La pensée des créateurs des grandes cultures, des Sumériens, des Egyptiens, des Grecs, etc., ne diffère pas dans sa création primitive des mots, de la pensée des Youkaguirs. Par rapport à ce terme, il existe une loi sémantique particulière établie par la linguistique japhétique - celle de la perception humaine commune $»^{8}$.

Dans ce passage, l'expression «perception humaine» a évidemment le sens de «pensée humaine », propre à des peuples «primitifs» : ce n'est pas pour rien si Marr utilise ici cet adjectif en parlant de la création des mots chez les Sumériens, les Egyptiens, les Grecs et les Youkaguirs. Ainsi le sens de cette loi sémantique contredit sa formulation et, tout en parlant de la perception humaine commune, c'est-à-dire, de la pensée qui serait la même à l'échelle mondiale, Marr, de façon beaucoup moins explicite que Lévy-Bruhl, distingue au moins deux types de pensée: primitive et l'autre, que, faute de terme marriste précis, nous appellerons pour l'instant non-primitive. Dans ce sens, Lévy-Bruhl était nettement un précurseur de Marr.

Alors, dans la première loi de la sémantique marriste, il ne s'agit guère de la perception humaine commune en général, mais de la pensée commune propre aux peuples appartenant à telle ou telle étape dans l'évolution de l'humanité. Ainsi, l'anthropologue français avec sa distinction explicite de deux types de pensée devient un «allié » idéal pour Marr, ce qui explique l'intérêt de ce dernier pour participer à la traduction de son livre en russe.

Et pourtant, qu'est-ce qui a fait que Marr a changé le titre du livre traduit en russe en 1930, ou, au moins, a accepté ce changement ? Que signifie ce jeu avec les mots primitif et inférieur?

Selon le contexte, l'adjectif français primitif peut correspondre à plusieurs adjectifs russes: pervobytnyj 'très ancien, préhistorique' (c'est le sens principal de ce mot russe); pervonačal'nyj, pervičnyj, isxodnyj 'originel, d'origine' et,

\footnotetext{
${ }^{7}$ Lévy-Bruhl, 1910, p. 37.

${ }^{8}$ Marr, 1933-1937, vol. III, p. 37.
} 
enfin, primitivnyj 'très simple, démodé'. A ce dernier sens correspond le mot français inférieur. Apparemment le traducteur (ou le rédacteur) du livre a essayé de se débarrasser de ce dernier sens dans la traduction russe du titre. Et pour réussir, il devait choisir un autre mot russe, pervobytnyj, dont le sens concernait avant tout des époques anciennes, préhistoriques - les époques auxquelles Marr consacrait l'essentiel de son attention. Ainsi la nécessité de remplacer un terme par un autre a exigé de remplacer un titre par un autre. Marr croyait toujours que le point le plus fort de sa «nouvelle théorie du langage » était précisément la possibilité de discuter les états préhistoriques de l'évolution langagière. En même temps, il reprochait au comparatisme son «attachement au temps historique » et son incapacité totale de dire quoi que ce soit sur les langues et le langage humain à l'époque préhistorique. C'est pourquoi, Marr ne reconnaissait qu'à contrecœur qu'«en réalité, chez LévyBruhl il ne s'agit pas de la vraie pensée primitive ${ }^{9}-$ et il est vrai que chez Lévy-Bruhl il ne s'agissait aucunement d'antiquités préhistoriques. L' anthropologue français décrivait les particularités d'un type spécifique de pensée qui était encore propre à certains de ses contemporains - les tribus des indigènes de l'Afrique, l'Australie, l'Océanie, etc. Cette situation était typique pour l'anthropologie du premiers tiers du XXème siècle, où les chercheurs se hâtaient de décrire un matériau qui semblait être en état de disparition.

Si Marr traçait des frontières entre les différents types de langues (les «stades » langagiers) dans le temps, les frontières de Lévy-Bruhl entre deux différents types de pensée concernaient l'espace, avant tout - bien qu'avec un présupposé sur l'évolution de l'humanité de l'un des types de pensée (la pensée primitive appartiendrait à la "préhistoire, alors que l'exigence logique était à peine sentie ${ }^{10}$ ) vers l'autre ${ }^{11}$.

Si l'anthropologue français distinguait deux stades (prélogique et logique) dans le développement de la pensée, Marr lui-même distinguait de trois à quatre étapes dans le processus glottogonique ${ }^{12}$ de l'évolution langagière, les langues correspondantes étant:

1) les langues du premier stade (le chinois, certaines langues africaines);

2) les langues du deuxième stade (le turc, le mongol, les langues finnoougriennes);

3) les langues du troisième stade - les langues japhétiques (certaines langues caucasiennes et sémitiques, ainsi que des langues dont l'origine restait encore inconnue à l'époque de Marr, comme par exemple le basque);

\footnotetext{
${ }^{9}$ Marr, 1930, p. XIV; nous soulignons.

${ }^{10}$ Lévy-Bruhl, 1910, p. 453.

${ }^{11}$ Cf. en particulier Lévy-Bruhl, 1910, p. 443.

12 Posant une loi générale et unique dans l'évolution de toutes les langues.
} 
4) les langues du quatrième stade - les langues indo-européennes qui, à la différence des langues appartenant aux trois groupes précédents, continuaient toujours de se développer.

Le fait que l'idée même des stades dans l'évolution des langues et de l'esprit humain ne date pas du début du XXème siècle a permis à l'historien de la linguistique russe V. Alpatov de considérer les travaux de Marr comme un «écho » des ouvrages des linguistes allemands du XIXème siécle, de W. von Humboldt et des frères A. et F. Schlegel ${ }^{13}$, avant tout.

Pourtant, malgré toute la ressemblance entre les travaux de ces linguistes et les théories de stades chez Marr, il y avait une grande différence: en réalité rien ne se répète exactement dans l'histoire des idées, et il semble que, en ce qui concerne les stades, les théories des linguistes soviétiques des années 1920-1930 avaient plus en commun avec les doctrines d'autres disciplines de cette époque qu'avec les conceptions linguistiques des époques précédentes. C'est pourquoi encore, la comparaison des théories de Marr et de LévyBruhl nous semble si importante.

Pour les linguistes allemands du XIXème siècle, les frontières entre les différents stades de l'évolution langagière étaient extérieures aux langues. En même temps, Marr et ses élèves pouvaient tracer les frontières entre les différents stades de l'évolution langagière à l'intérieur des langues elles-mêmes. Cela signifie que, pour Marr, dans chaque langue et à chaque stade de son évolution, on pouvait discerner, à plusieurs niveaux, les éléments des stades précédents, des «couches» appartenant aux différents $\operatorname{stades}^{14}$. Au niveau phonétique, en particulier, c'était la possibilité de faire remonter tous les mots de toutes les langues aux «quatre éléments primitifs » (sal, jon, ber, roš) qui, selon Marr, avaient remplacé le langage gestuel et étaient à l'origine du langage sonore, en constituant le langage des magiciens, la caste au pouvoir ${ }^{15}$. Au niveau syntaxique, ce sont les constructions impersonnelles dans les langues modernes que Marr considérait comme vestiges du passé des langues.

Dans toutes ses réflexions, les «traces du passé » dans la langue sont liées aux formes linguistiques : il peut s'agir de formes en tant que telles (les traces des quatre éléments primitifs dans les formes des signes linguistiques des langues modernes) ou des rapports formes $v s$. contenus, si les formes ne correspondent plus aux contenus (par exemple, les anciennes constructions syntaxiques se rempliraient de nouveaux sens et en disant aujourd'hui Il fait froid nous ne penserons plus aux «êtres mythologiques » qui nous feraient froid - ce qui aurait été le cas jadis, selon Marr). Il s'ensuit que, selon Marr,

\footnotetext{
${ }^{13}$ Alpatov, 1991, p. 121.

${ }^{14}$ Marr, 1933-1937, vol. II, p. 455.

${ }^{15} \mathrm{Ibid}$., vol. III, p. 329.
} 
au cours de l'évolution la forme linguistique changerait beaucoup moins vite que le contenu.

Les idées marristes sur les frontières entre les stades, qui étaient intérieures par rapport aux différentes langues (c'est-à-dire, dans la même langue on pouvait distinguer les éléments de différents stades) avaient sûrement beaucoup en commun avec les idées de Lévy-Bruhl sur l'absence de frontières strictes entre les deux types de pensée - prélogique et logique. La mentalité des sociétés inférieures, tout en devenant moins imperméable à l'expérience, demeurerait longtemps prélogique et garderait l'empreinte mystique sur la plupart de ses représentations. En outre, rien ne préserverait les concepts abstraits et généraux, une fois formés, de retenir des éléments qui sont des vestiges encore reconnaissables de la période antérieure ${ }^{16}$.

En revenant à l'histoire de la traduction russe et à la substitution d'un titre par un autre, nous pouvons maintenant supposer que, n'étant pas pris au sérieux par la plupart des linguistes indo-européistes de l'Occident, contre les théories desquels il s'était élevé dès le début de sa carrière scientifique, Marr voulait probablement se trouver des alliés étrangers parmi les représentants d'autres disciplines. Lévy-Bruhl devait être l'un de ceux-là. Mais pour le présenter en tant qu' «allié » en URSS, il fallait au moins prétendre qu'il écrivait sur le même sujet que l'auteur de la «nouvelle théorie du langage». C'est pourquoi, l'adjectif inférieur (qui n'a aucune allusion à la préhistoire marriste) a été remplacé par le mot primitif (où cette allusion est tout à fait claire) dans le titre russe - et donc un titre a été remplacé par un autre.

Loi des opposés et loi de la sémantique diffuse. Langue ou langage?

(Formes sans contenus et contenus sans formes)

La loi des opposés (zakon protivopoložnostej) formulée par Marr consiste en la divergence du sens originel et 《diffus $»^{17}$ en deux sens opposés, plus concrets:

«Selon la loi de la division sémantique, à l'origine la même base servait à exprimer deux sens opposés $»^{18}$.

En parlant de cette loi, Marr était très avare d'exemples. En voici néanmoins quelques-uns:

\footnotetext{
${ }^{16}$ Lévy-Bruhl, 1910, p. 447.

${ }^{17}$ Marr ne définit jamais ce qu'il sous-entend par « diffus ». Faute de mieux, définissons pour l'instant "diffus » dans le domaine de la sémantique comme polysémique à un très haut degré. En réalité, comme nous le verrons, les rapports entre les catégories de «diffus » et de «polysémique » chez Marr sont encore plus complexes.

${ }^{18}$ Marr, 1933-1937, vol. III, p. 18.
} 
1) à l'époque préhistorique, 'bon' présupposait en même temps 'mauvais ${ }^{19}$,

2) le 'début' et la 'tête' — la 'fin' et la 'queue ${ }^{\text {,20, }}$

3) le 'jour' et 'blanc' - la 'nuit' et 'noir',21,

4) le 'haut' - le 'bas',22,

5) le 'ciel' - la 'terre, ${ }^{, 23}$, etc.

La loi sémantique de la divergence du sens primitif en deux sens opposés a été longtemps considérée comme l'un des grands mérites de Marr dans le domaine de la sémantique. Il est vrai qu'à première vue, la loi des opposés semble un point très original de la doctrine marriste. Néanmoins, déjà avant la théorie marriste, non seulement en Russie, mais aussi dans d'autres pays, d'autres théories semblables étaient apparues, qui partaient du principe de la division sémantique des mots au cours de l'évolution des langues. Entre autres, on trouvait du matériau pour «prouver» ces théories dans les cas illustrant le phénomène qui révèle l'existence de «mots opposés » (l'énantiosémie) et on considérait que ce phénomène était propre, avant tout, auxAaingiuescdmoinomiquement, Marr et les marristes étaient plutôt les derniers que les premiers chercheurs à donner à ce phénomène une explication liée à l'évolution du langage et de la pensée.

Dans la doctrine marriste, la loi des opposés semble être très proche d'une autre loi sémantique qui est plus générale - même si explicitement Marr ne la formule pratiquement jamais. Il s'agit de la loi de la divergence sémantique en plusieurs sens, ou loi de la «sémantique diffuse» — c'est nous qui la désignons ainsi, faute de formulation de Marr lui-même, qui, en ce cas, n'appelle explicitement «lois » que les manifestations particulières de cette loi. Cette loi suppose l'évolution sémantique de tous les mots dans toutes les langues à partir de «séries » (rjady), «faisceaux » (pučki) ou «nids » (gnezda) qui réunissaient plusieurs sens. La divergence sémantique, selon Marr, signifie la division de ces «nids» sémantiques en des sens plus concrets. Voici l'un des exemples:

«La paléontologie de la parole nous montre que le 'poisson', aussi bien que la 'pluie', a reçu son nom de l' 'eau'. Quant à la sémantique de ces mots, le chinois garde toujours cet état primitif, quand on employait le même mot signifiant l' 'eau' pour dire 'la pluie' et 'le poisson' ${ }^{24}$.

\footnotetext{
${ }^{19}$ Ibid., p. 14.

${ }^{20}$ Ibid., vol. III, p. 96.

${ }^{21} \mathrm{Ibid}$.

${ }^{22}$ Ibid., vol. III, p. 96.

${ }^{23}$ Ibid., vol. II, p. 220.

${ }^{24}$ Ibid., p. 55.
} 
Ici l' 'eau' est un «nid» sémantique qui réunirait des sens tels que 'poisson' et 'pluie'. A la différence de la loi des opposés où il était très avare d'exemples, Marr donne dans ses articles de très nombreux exemples de « nids » sémantiques, et leur nombre dépasse de loin sept.

Pourtant, Marr ne s'arrête pas là et il fait remonter tous les sens existant dans les langues d'aujourd'hui à un seul sens originel. Il s'agit du «'ciel', le nid des proto-sens », selon le titre de son article datant de $1923^{25}$.

Aussi bien, dans ses autres travaux Marr parle explicitement de l' existence d'un seul mot à l'origine du langage:

«Le langage sonore a plusieurs dizaines de milliers d'années. Il suffit de dire qu'aujourd'hui la paléontologie linguistique nous donne la possibilité d'atteindre l'époque où les tribus n'avaient qu'un seul mot et l'utilisaient dans tous les sens dont l'humanité se rendait compte à cette époque $»^{26}$.

Même si dans cet article Marr ne dit pas quel était ce «mot» «polysémique $»^{27}$ primitif, ses autres travaux ne laissent aucune doute - il s'agissait bien du 'ciel' :

« [Au début], chaque tribu primitive n'avait qu'un seul mot, qui était le totem et le dieu. Le 'ciel' était le premier totem, [...] il constituait l'image centrale et il a donné naissance à un grand nombre de chaînes sémantiques, c'est-à-dire, à des séries de sens liés les uns avec les autres ${ }^{28}$; «en prononçant le mot 'ciel', je l'utilise conventionnellement tel qu'il se présentait à la pensée rudimentaire de l'homme primitif, qui identifiait 'le ciel' avec tout l'univers, y compris lui-même, il se représentait 'le ciel' comme composant de tous les éléments, avant tout, de 'l'eau' (plus 'l'obscurité'), et donc, comme nous verrons, de son antithèse, 'le feu' (plus 'la lumière') »'.

Le principe-clé de ces deux lois de la sémantique marriste est celui de divergence. Ce même principe était à la base des théories qui concernaient d'autres niveaux linguistiques (en particulier, la syntaxe: une phrase « diffuse » et «non-divisée » serait à l'origine de la syntaxe moderne, et la phonétique: les célèbres quatre éléments « diffus » auraient donné la naissance à tous les sons de toutes les langues modernes) et étaient élaborées par les collègues et les élèves de Marr ou par les linguistes qui n'étaient pas marristes, mais qui se référaient dans leurs théories à l'autorité de Marr ${ }^{30}$.

\footnotetext{
${ }^{25}$ Ibid., p. 143-146.

${ }^{26}$ Ibid., vol. I, p. 217.

${ }^{27}$ Plus tard, nous expliquerons la nécessité de prendre ces mots entre guillemets.

${ }^{28}$ Marr, 1933-1937, vol. I, p. 213.

${ }^{29} \mathrm{lbid}$., vol. II, p. 207.

${ }^{30} \mathrm{Cf}$. Velmezova, 2005b. A première vue, il semble que les fondements philosophiques et épistémologiques de toutes ces théories évoquant la divergence au cours de l'évolution langagière soient les
} 
Après avoir découvert quels étaient les «proto-formes » (les quatre éléments primitifs) et les «proto-sens » (les «nids » sémantiques) chez Marr, posons la question suivante: quels étaient les rapports entre les deux ? En d'autres mots, que signifiait chacun des quatre éléments et par quelles formes les «nids » sémantiques étaient-ils désignés ?

La réponse semble paradoxale: la théorie marriste n'établissait aucune correspondance entre ces proto-formes et ces proto-sens. Cela s'ensuit déjà de la différence quantitative des éléments primitifs sémantiques et formels: il y aurait quatre complexes sonores primitifs (sal-ber-jon-roš) et un seul proto-nid sémantique ('ciel'). Ainsi Marr réfléchissait au sujet des proto-formes et des proto-sens sans établir de lien entre eux, ce qui contredit en principe toutes les tentatives de reconstruction de la proto-langue à l'époque de Marr et avant lui. Le processus même de cette reconstruction présupposait qu'il fallait mettre en correspondance certaines formes et des contenus particuliers.

mêmes. Et pourtant, il nous semble qu'il serait erroné de confondre deux principes différents: celui de la division des «éléments primitifs » en deux éléments et celui de leur divergence en plusieurs parties. Dans le premier cas, la loi des opposés chez Marr semble être liée à la doctrine de G.-W.-F. Hegel sur le «concept » exprimant le fondement de toutes les choses, auquel Hegel a consacré une grande partie de sa Wissenschaft der Logik. Le concept (Begriff) ou l'idée (Idee), selon Hegel, exprime l'état embryonnaire de la chose qui, ensuite, se différencie et se réalise graduellement. $A$ une étape primitive de la connaissance, la définition de l'objet dans l'idée n'est que très générale et abstraite. Elle se concrétise petit à petit, et au cours de la connaissance, la différenciation évolue et commence à être exprimée, en passant vers des objets de plus en plus concrets. La thèse générale exprimée dans les travaux de jeunesse de Hegel et qui, en principe, a formé la base de sa dialectique - énonce la transformation dialectique de l'unité primitive de la vie en son contraire, divisé en deux parties. En dépassant cette division, nous revenons encore à l'unité, cette fois plus riche et concrète. Ainsi les contradictions sont considérées comme une source intérieure du développement, telle une « montée » de l'abstrait vers le concret. D'un autre côté, les idées des marristes sur l'évolution du « diffus » et sa divergence en plusieurs parties sont beaucoup plus proches des théories de H. Spencer, pour qui l'évolution consiste en transformation de l'homogène en hétérogène (cf. en particulier Spencer, 1907, p. 32). Spencer considérait cette loi de la différenciation de la matière physique (biologique, avant tout) comme universelle et essayait de l'appliquer aux différentes branches des sciences humaines: l'histoire de la société, la religion, la psychologie. Ainsi entre 1862 et 1896, Spencer a créé un système de philosophie synthétique, et les idées des marristes analysées dans cette partie de notre travail peuvent être considérées comme une application de la philosophie de Spencer en linguistique. Bien sûr, il ne s'agit pas d'une influence directe ou même consciente, mais plutôt de l'air du temps dans lequel ces idées apparaissaient et se développaient. En particulier, Marr ne cite jamais Spencer - bien que, dans ses notes non-publiées il avoue qu'encore au gymnase il ait lu et relu maintes fois les travaux de Spencer. Quant à Hegel, le «précurseur du marxisme » si aimé par les chefs soviétiques, Marr ne le mentionne que dans un seul article de ses CEuvres choisies (Marr, 1933-1937, vol. II, p. 444-459) - pourtant, ses travaux où il s'agit de la loi des opposés, semblent en être imprégnés (comme, d'ailleurs, pratiquement toute la culture intellectuelle russe à la charnière des siècles. Sur la composante hégélienne dans la pensée des intellectuels russes au XIXème siècle cf. en particulier Čiževskij, 1939).

26 VOLUME 2, NÚMERO 2, 2006 
Voilà la réponse à la question de savoir pourquoi la notion de sens diffus en principe ne peut pas être définie par celle de polysémie. Les dictionnaires d'aujourd'hui définissent le phénomène de polysémie en synchronie comme « caractère d'un signe qui, à fonction constante (verbe, nom, etc.) possède plusieurs signifiés à noyau commun $»^{31}$. Ainsi ce n'est qu'une forme linguistique qui peut être polysémique, tandis que dans le cas de la sémantique diffuse, nous n'en avons aucune. C'est pourquoi encore, on ne peut point dire que chez Marr le premier mot du langage humain signifiait 'ciel': il ne s'agit pas du mot, car le sens 'ciel' n'était attaché à aucune forme linguistique particutidìnactère «parallèle » des réflexions de Marr au sujet des proto-formes et des proto-sens se révèle clairement dans les thèses marristes (qui ne sont pas explicites, mais se laissent reconstruire) sur leur évolution. En fait, si la sémantique des langues change (les «nids » sémantiques n'existent plus dans la plupart des langues modernes), le côté formel des langues, au contraire, ne change pas ou change très peu, et tous les mots de toutes les langues modernes, selon Marr, recèlent toujours les quatre éléments de départ.

La thèse générale des marristes sur le caractère archaïque des éléments «diffus », par rapport aux éléments «concrets » est très importante pour comprendre la conception évolutionniste de Marr en général. En fait, Marr présentait le schéma de l'évolution langagière à l'inverse de celui des langues particulières $^{32}$. Selon lui, ces dernières ne se développaient pas depuis une unité primitive particulière («langue-mère ») vers une multiplicité (un axiome de l'indo-européanisme classique, à commencer par A. Schleicher, adepte de Ch. Darwin en linguistique). C'est A. Schleicher qui a introduit en linguistique la notion actuellement très répandue de l'arbre généalogique des langues, expliquant l'évolution des langues de l'unité primitive vers la multitude. En revanche, chez Marr un schéma semblable pouvait être appliqué à l'évolution du langage qui était donc proclamée divergente, tandis que les langues, au contraire, se développaient de la multiplicité vers l'unité, par convergence (hybridation) $^{33}$.

Les schémas suivants présentent l'évolution des langues selon Marr et selon les comparatistes traditionnels (à commencer par Schleicher), aussi que l'évolution du langage chez Marr :

Schéma 1. Le développement des langues, selon les comparatistes traditionnels et selon Marr

\footnotetext{
${ }^{31}$ GRLF, 2001, p. 925.

${ }^{32}$ Pour distinguer explicitement les deux dans la conception marriste, on peut s'appuyer sur la distinction langue / langage proposée par de Saussure (Saussure, 1916 [1983, p. 25]).

${ }^{33}$ Marr, 1933-1937, vol. II, p. 135.
} 
Le schéma des comparatistes traditionnels:

langue-mère
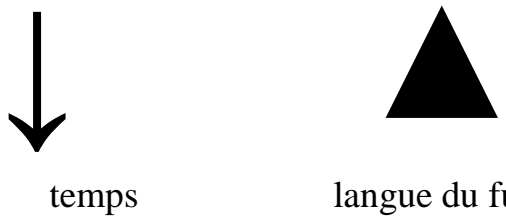

langue du futur

Le schéma de Marr:

langue-mère
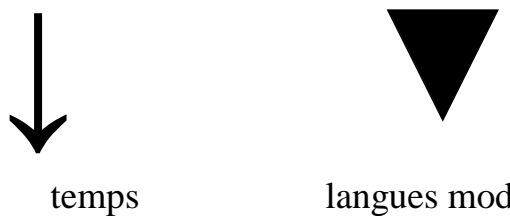

temps

langues modernes

Schéma 2. Le développement du langage, selon Marr

l'état « protolangagier », « diffus »
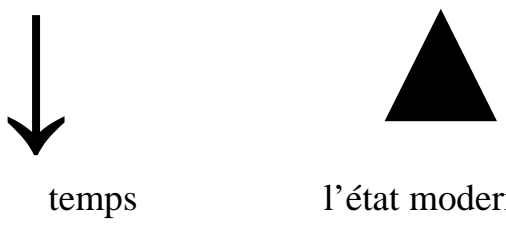

l'état moderne

Marr lui-même ne distinguait pas explicitement le langage et la langue: en russe, il n'y a qu'un seul mot (jazyk) pour désigner les deux. Pourtant, cette opposition (qui prend forme à partir du Cours de linguistique générale de $\mathrm{F}$. de Saussure) sert à comprendre de nombreuses thèses et principes marristes qui semblent incohérents à première vue. En particulier, comment peut-on expliquer, sans introduire cette opposition, l'affirmation de Marr sur la dérivation de tous les mots de toutes les langues modernes à partir des quatre éléments primitifs et, en même temps, sa thèse sur la multitude des langues à l'origine ? Ces deux affirmations semblent contradictoires et incompatibles quand on lit Marr en russe et pourtant, dans le premier cas il s'agit du langage, tandis que dans le deuxième cas - des langues particulières.

28 VOLUME 2, NÚMERO 2, 2006 
Ainsi, de nombreuses «contradictions » dans les travaux de Marr «disparaissent » dans la traduction de ses travaux du russe dans une langue comme le français, dans laquelle, à la différence du russe, les notions de 'langue' et de 'langage' sont exprimées par des mots différents.

Ces « contradictions » de Marr ont beaucoup contribué à l'incompréhension de ses doctrines, quand, en particulier, on considérait ses théories comme révélant la doctrine monogénétique de l'origine des langues ${ }^{34}$.

Or, si nous nous reportons aux schémas proposés plus haut, nous voyons que la célèbre thèse marriste sur les quatre éléments primitifs ne contredit en aucune façon son principe de l'origine polygénétique des langues: dans le premier cas, il s'agit du langage, tandis que dans le deuxième cas - des différentes langues.

\section{Loi de la transposition fonctionnelle: à propos de l'intervention de Staline en linguistique (Les signes linguistiques seraient-ils arbitraires?)}

Des centaines de pages ont déjà été écrites sur l'intervention de Staline en linguistique en 1950. La plupart des analystes se sont donné pour but la présentation et l'analyse de la discussion linguistique en 1950 au vu des événements de la vie politique et sociale en URSS. Pour notre part, nous essaierons de concentrer notre attention sur l'analyse du contenu proprement linguistique de cette intervention, pour comprendre ce que cette action politique signifiait pour la linguistique.

Après l'article du dictateur publié dans la Pravda du 20 juin 1950, ses réponses «aux lettres des lecteurs » ont été publiées dans les périodiques Bolševik (29 juin 1950) et Pravda (4 juillet et 2 août 1950). L'article du 20 juin a été composé sur le modèle d'un dialogue avec «un groupe de jeunes camarades ». Staline se donnait pour but de répondre aux quatre questions suivanfest:il vrai que la langue est une superstructure au-dessus d'une base $?^{35}$ (Staline a donné une réponse négative à cette question);

- Est-il vrai que la langue a toujours porté et porte un caractère de classe, qu'il n'existe pas de langue commune et unique pour la société, langue dépourvue d'un caractère de classe, mais appartenant au peuple tout entier? ${ }^{36}$ (la réponse était aussi négative);

- Quels sont les signes caractéristiques de la langue? ${ }^{37}$ (dans ses raisonnements sur l'évolution des langues, Staline de facto revenait aux

${ }^{34}$ Phillips, 1986, p. 78; Slodzian, 1994/1995, p. 123; Čikobava, 1985, p. 15, etc.

${ }^{35}$ Staline, 1950 [1977, p. 147].

${ }^{36}$ Ibid., p. 154

${ }^{37}$ Ibid., p. 166. 
conceptions des néo-grammairiens qui reconnaissaient, à la différence des marristes, la parenté des langues);

- La Pravda a-t-elle eu raison d'ouvrir une discussion libre sur les questions de linguistique? ${ }^{38}$

Il est probable que cet ordre de questions correspondait à leur degré d'importance pour Staline (en fait, il n'avait même pas besoin de répondre à la dernière question: bien sûr, la Pravda a eu raison d'ouvrir cette discussion, si Staline en personne décida d'y participer!).

En somme, dans ses réponses, Staline présentait ses opinions sur le caractère de l'influence mutuelle des langues dans leur évolution, aussi bien que sur la nature même de la langue.

Aussi paradoxal que cela puisse paraître, la vision générale du premier problème par Staline ne contredisait pas toutes les conceptions marristes même si en grande partie, le dictateur soviétique est revenu dans sa critique au modèle «génétique » des néo-grammairiens en linguistique.

Les désaccords principaux entre Marr et Staline concernaient plutôt leurs opinions sur la nature de la langue. En s'élevant contre la considération de la langue parmi les catégories de la superstructure aussi bien que contre la surestimation des études sémantiques, Staline rejetait un concept très important introduit en linguistique par les marristes: il s'agit de la notion de sémantique idéologique, qui remonte aux notions de sémantique et d'idéologie chez Marr et demande aujourd'hui l'étude la plus poussée.

Dans les années 1920, Marr lie l'idéologie à la superstructure sociale, dont l'évolution dépend de l'infrastructure. Comme la langue pour lui fait partie de la superstructure, l'idéologie de chaque société aurait toujours son reflet linguistique. Et c'est la sémantique de la langue qui reflète, avant tout, l'idéologie de la société en question - en grande partie, parce que, selon Marr, ce n'est que la sémantique qui est flexible, tandis que la forme linguistique change beaucoup moins. La dépendance de la sémantique de l'évolution de l'infrastructure est reflétée dans la loi de la transposition fonctionnelle, considérée comme le mérite particulier de la doctrine marriste. Dans cette loi, il s'agissait de la transposition du nom de l'objet sur un autre, qui joue le même rôle que le premier objet dans la société à un nouveau stade de sAimnśsiopoutriouxemple, si certains peuples avaient mangé les glands avant d'apprendre à cultiver le blé, l'apparition de l'agriculture serait pour eux le moment où les noms signifiant les glands ont été transposés sur ce que nous appelons aujourd'hui les produits panifiés ${ }^{39}$; l'or a reçu son nom de la

\footnotetext{
${ }^{38}$ Ibid., p. 175

${ }^{39}$ Marr, 1933-1937, vol. IV, p. 151.
} 
fourrure, autrefois symbole de la richesse dans la sociétée ${ }^{40}$; «le 'père' (l' 'homme') a reçu son nom de la 'mère' (la 'femme'), [...] car le père a obtenu la fonction de mère dans la conscience humaine ${ }^{41}$, après le passage du matriarcat au patriarcat, etc.

Après la mort de Marr, la notion de sémantique idéologique a été développée dans les travaux de ses élèves et collègues - V.I. Abaev (19002001), essentiellement. Selon V. Abaev, la forme linguistique est toujours technique. La sémantique, par contre, peut être soit technique, soit idéologique. Voici comment il établit cette distinction :

«Le linguiste-historien peut étudier la sémantique du mot de deux façons différentes: d'une part, la sémantique en tant que minimum des fonctions sémantiques qui déterminent l'emploi moderne et communicatif du mot. C'est la "petite sémantique" qu'on peut également définir comme la sémantique "technique". D' autre part, la sémantique en tant que somme de toutes les représentations cognitives et émotionnelles accompagnantes, dans lesquelles la vie intérieure et complexe du mot se reflète dans son passé et dans son présent. C'est de la "grande sémantique", pour laquelle nous avons proposé $^{42}$ le terme de "sémantique idéologique" ${ }^{43}$.

Si la «petite sémantique » du mot est reflétée dans les dictionnaires, la « grande sémantique » du mot peut être présente de deux manières différentes.

Premièrement, dans l'étymologie du mot. Deuxièmement, la sémantique idéologique du mot se découvre dans ses liens avec d'autres mots, qui ne doivent pas nécessairement être liés étymologiquement avec le premier. La vision particulière de ce problème par Marr se manifestait dans l'établissement de liens sémantiques et étymologiques tout à fait fantaisistes entre les mots, à partir de la simple ressemblance de leurs formes. Par exemple, Marr établissait des liens entre les mots allemands Hund et hundert, en proposant la chaine sémantique suivante : 'le chien en tant que totem' $\rightarrow$ 'le nom du collectif' $\rightarrow$ 'tous' $\rightarrow$ 'beaucoup' $\rightarrow$ 'cent' ${ }^{44}$.

Nous avons déjà vu que l'explication des vestiges dans les langues chez Marr était liée au fait que le contenu évoluerait plus vite que la forme linguistique. Ainsi la forme, à la différence du contenu, pourrait être liée à l'étymologie qui refléterait une idéologie ancienne. Par exemple, les liens sémantiques (et donc, pour Marr, idéologiques) entre Hund et hundert ne seraient pas actuels, mais liés à une époque ancienne, à l'époque où le chien

\footnotetext{
${ }^{40}$ Ibid., vol. V, p. 198.

${ }^{41}$ Ibid., vol. IV, p. 194.

${ }^{42}$ Cf. Abaev, 1934.

${ }^{43}$ Abaev, 1948, p. 15.

${ }^{44}$ Marr, 1933-1937, vol. II, p. 391.
} 
aurait été le totem d'une tribu. Ainsi la forme linguistique nous donnerait la clé de l'étymologie qui, à son tour, serait révélatrice d'une ancienne sémantique.

La plupart des étymologies de Marr étaient fantaisistes du point de vue de l'étymologie moderne, ce que même ses collègues reconnaissaient ${ }^{45}$. Pourtant, du point de vue de Marr lui-même, il n'y avait pas de fautes, car ses études sémantiques et étymologiques reposaient sur d'autres principes que ceux des étymologistes d'aujourd'hui: avant tout, sur la thèse anti-saussurienne de la dépendance du contenu de la forme linguistique.

On peut comparer son approche avec le principe de l'étymologie populaire. Ce dernier présuppose l'établissement de liens sémantiques entre des mots dont les formes sont semblables. En d'autres termes, la célèbre thèse saussurienne sur le caractère arbitraire des liens entre le signifiant et le signifié (« le lien unissant le signifiant au signifié est arbitraire ${ }^{46}$ ) est contestée, car l'interprétation du contenu du mot devient directement dépendante de sa forme.

La négation de la thèse de Saussure chez Marr est sûrement liée au principe d'attribuer une valeur révélant une idéologie particulière non seulement au côté sémantique de la langue, mais aussi à son côté formel, ses sons, ses phonèmes et même les lettres de son alphabet. Même si ce principe ne présuppose pas la dépendance nécessaire du contenu de la forme linguistique, les liens entre la forme et le contenu y sont quand même beaucoup plus manifestes que dans la conception saussurienne.

Comme on peut le voir, la sémantique, liée à l'idéologie de la société, évoluerait plus vite que la forme linguistique. A son tour, la forme linguistique serait aussi idéologique, mais «avec du retard», étant révélatrice des idéologies anciennes. C'est pourquoi, l'étymologie à base formelle permettrait de reconstruire la sémantique idéologique du passé.

En revenant à la critique stalinienne de la sémantique marriste, posons la question suivante: pourquoi Staline a-t-il choisi la notion de sémantique idéologique en tant qu'objet principal de sa critique ? De toute évidence, le contenu de cette notion ne devait pas correspondre à sa politique dans les années 1950. Plus exactement, il s'agissait de l'idée sur la division de la nation en différentes classes, implicitement liée au fait de considérer la langue parmi les phénomènes de superstructure. Dans les années de la guerre (1941-1945) et de l'après-guerre, les objectifs principaux de la politique intérieure et extérieure soviétique (le refoulement des troupes allemandes et, ensuite, la reconstruction du pays) demandaient de l'unité idéologique de la nation. C'est pourquoi, l'idée même d'une division quelconque (y compris sociale) était insupportable pour Staline.

${ }^{45}$ En particulier, Abaev estimait les erreurs dans tous les exemples linguistiques chez Marr à environ 75\% (Abaev, 1948, p. 14).

${ }^{46}$ Saussure, 1916 [1983, p. 100].

32 VOLUME 2, NÚMERO 2, 2006 
Or comme chaque classe a sa place dans l'infrastructure de la société (par rapport aux outils de production) et comme chez Marr la langue est un phénomène de superstructure qui dépend de l'infrastructure, chaque classe devrait avoir sa propre langue ${ }^{47}$. A son tour, le principe de la division (sociale et linguistique) de la nation et d'absence de toute langue nationale devait être inacceptable pour Staline après la Seconde guerre mondiale, ce qui a provoqué son opposition radicale aux doctrines marristes.

\section{EN GUISE DE CONCLUSION}

Même si Marr prétendait souvent le contraire, la sémantique en tant que sous-branche de la linguistique existait avant le marrisme - même si au XIXème siècle la plupart des linguistes lui prêtaient moins d'attention qu'à la phonétique ou à la syntaxe. La notion de loi sémantique en diachronie existait bien avant la «nouvelle théorie du langage». De plus, les « découvertes » de Marr qui étaient au centre de ces quatre lois sémantiques ont été également discutées par d'autres chercheurs, aussi bien avant Marr qu'à son époque, en Russie et dans d'autres pays.

Si nous pouvons constater une certaine originalité de Marr quant à son approche des problèmes sémantiques, elle n'était pas dans ses propres déclarations, mais plutôt dans les principes de base de ses théories, qu'il n'avait pourtant jamais explicitement exprimés et que nous sommes obligés de chercher, de reconstruire aujourd'hui «entre les lignes » de ses nombreux travaux. Notre l'analyse des quatre lois sémantiques de Marr a permis de découvrir plusieurs principes-clés du marrisme - qui, encore une fois, ne sont jamais explicités chez lui. D'une part, il s'agit de la distinction nette entre les notions de langue et langage, dont l'importance est primordiale pour comprendre la conception marriste de l'évolution en linguistique et sa théorie de la polygenèse des langues évoluant par convergence, à la différence du langage qui évolue par divergence, à partir des éléments prinfletifistex difftssce qui nous semble encore plus important, de nombreuses « aberrations » et «contradictions », voire «obscurités » de Marr disparaissent à la lumière du principe établissant des relations très spécifiques entre la forme et le contenu des signes linguistiques - bien qu'à première vue ce soit précisément cette partie de la doctrine marriste qui semble la plus contradictoire et pleine de paradoxes: les formes linguistiques privées de sens, les contenus sans formes, les formes qui sont en retard par rapport aux contenus ou qui s'arrêtent dans leur évolution. Il se trouve que les notions de

${ }^{47}$ Marr, 1933-1937, vol. II, p. 415. 
sens et de forme avaient chez les marristes un tout autre contenu que celui auquel nous sommes habitués après F. de Saussure.

Ainsi, en ce qui concerne la diachronie, les liens entre les formes et les contenus seraient faibles, voire inexistants. Pour l'état préhistorique du langage humain, Marr établit la coexistence des sens et des formes linguistiques diffus qui n'auraient aucun lien les uns avec les autres. Dans l'évolution linguistique, les formes et les contenus se développeraient à des vitesses différentes, de façon que les formes seraient toujours en retard par rapport aux contenus, directement liés à l'idéologie de la société. Cela explique les survivances, les traces du passé dans les langues modernes. D'autre part, liées aux étymologies, les formes permettraient la reconstruction de la sémantique du passé. Cette composante du marrisme a certainement influencé l'évolution de la linguistique en URSS après la mort de Marr et un travail reste encore à faire pour comparer ce qui semble incomparable, pour établir des rapports entre des chercheurs et des courants linguistiques qui, à première vue, semblent n'avoir rien en commun.

\section{RÉFÉRENCES BIBLIOGRAPHIQUES}

ABAEV, Vasilij Ivanovič. Jazyk kak ideologija i jazyk kak texnika. Jazyk i myšlenie, Leningrad, vol. II, 1934, p. 33-54. [La langue comme idéologie et la langue comme technique] . Ponjatie ideosemantiki, Jazyk i myšlenie, Moskva-Leningrad, vol. XI, 1948, p. 13-28. [La notion de sémantique idéologique]

ALPATOV, Vladimir Mixajlovič. Istorija odnogo mifa, Marr i marrizm. Moskva, Nauka, 1991. [L'histoire d'un mythe. Marr et le marrisme].

ČIKOBAVA, Arnol'd Stepanovič. Kogda i kak eto bylo?, Ežegodnik iberijsko-kavkazskogo jazykoznanija, Tbilisi, vol. XII, 1985, p. 9-23. [Quand et comment cela s'est passé ?]

ČIŽEVSKIJ, Dmitrij Ivanovič. Gegel' v Rossii. Paris, Dom knigi i Sovremennye zapiski, 1939. [Hegel en Russie]

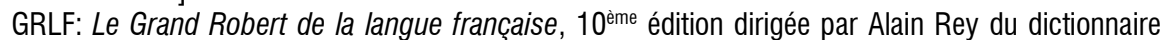
alphabétique et analogique de la langue française de Paul Robert. Paris, Dictionnaires le Robert, 2001, tome 5.

LEVI-BRJUL' (Lévy-Bruhl), Ljis'en. Pervobytnoe myšlenie. Moskva-Leningrad, Ateist, 1930. [La mentalité primitive].

LEVY-BRUHL, Lucien. Les fonctions mentales dans les sociétés inférieures. Paris, F. Alcan, 1910.

MARR, Nikolaj Jakovlevič. Predislovie k knige L. Levi-Brjulja Pervobytnoe myšlenie, in LEVIBRJUL', 1930, p. XIV-XV. [Préface au livre de L. Lévy-Bruhl La mentalité primitive]

Izbrannye raboty. Vol. I-V, Moskva-Leningrad, Izdatel'stvo gosudarstvennoj akademii istorii material'noj kul'tury (vol. I), Gosudarstvennoe social'no-èkonomičeskoe izdatel'stvo (vol. II-V), 1933-1937. [CEuvres choisies].

PHILLIPS, Katherine H. Language Theories of the Early Soviet Period, Exeter Ling. Studies, University of Exeter, 1986.

SAUSSURE, Ferdinand de. Cours de linguistique générale. Paris, Payot, 1983 [1916].

34 VOLUME 2, NÚMERO 2, 2006 
SLODZIAN, Monique. Slodzian, La doctrine terminologique, nouvelle théorie du signe au carrefour de l'universalisme et du logicisme, ALFA, 1994/1995, vol.7/8, p. 121-136.

SPENCER, Herbert. Les premiers principes. Paris, Félix Alcan, 1907.

STALINE, Iosif Vissarionovič. A propos du marxisme en linguistique (article publié dans le journal Pravda le 20 juin 1950) [1950], in Calvet L.-J. (éd.) Marxisme et linguistique. Paris, Payot, 1977, p. 145-196.

VELMEZOVA, Ekaterina. Lucien Lévy-Bruhl lu par Nikolaj Marr: deux théories des langues dites primitives, Slavica Occitania, Toulouse, № 17 Entre Russie et Europe: itinéraires croisés des linguistes et des idées linguistiques. 2003, p. 119-138.

'Primitif' ou 'inférieur' ? Une traduction « marriste » de L. Lévy-Bruhl en russe, The Henry Sweet Society for the History of Linguistic Ideas Bulletin, № 45, 2005, p. 43-51.

$\checkmark$ načale byla... diffuznost'? (0 filosofsko-èpistemologičeskix predposylkax nekotoryx èvoljucionistskix teorij v lingvistike v konce XIX - načale XX v.), Jazyk. Ličnost’. Tekst. Sbornik statej k 70-letiju T.M. Nikolaevoj. Moskva, Jazyki slavjanskoj kul'tury, 2005, p. 73-86. [Au début était... le diffus ? (Sur les prémisses philosophiques et épistémologiques des certains théories linguistiques à la fin du XIXème - début du XXème siècle)] 